\title{
SENSORY-INTEGRATION THERAPY IN THE TREATMENT OF AGGRESSIVE BEHAVIOR IN CHILDREN WITH MULTIPLE DISABILITIES
}

\author{
Diyana Georgieva ${ }^{1}$
}

\begin{abstract}
In recent years, sensory-integration therapy has emerged as one of the most popular treatments for aggressive behavior in children with different disorders. This article is devoted to an experimental study aimed at determining the effects of sensory-integration therapy on the aggressive behavior of children with multiple disabilities. Eighteen children participated in the study. The registration of the children's behavioral responses for 24 consecutive school days allowed the collection of evidence in support of the thesis in the study. The individual child's frequency of aggressive behaviors in four domains was recording on 12 days while undergoing sensory-integrative therapy and 12 days without a treatment with sensory-integrative techniques. The following methods were used in the analysis of the results: Paired samples t-tests to test the four null hypotheses, a Shaprio-Wilk test for the assumption of normality, Cohen's d to determine the effect size attributable to the therapy, and descriptive statistics for the dependent variables. The statistical results indicated a high level of significance for the three variables: aggression towards property, physical aggression, and aggression toward self. The obtained average values for the three modules are evidence for the positive effects of the sensory-based therapy in children with emotional and behavioral problems. The fourth variable, verbal aggression did not show a statistically significant difference between the mean values before and after the sensory-based intervention.
\end{abstract}

UDC Classification: 376, DOI: https://doi.org/10.12955/pss.v2.211

Keywords: sensory-integration therapy, treatment, children with multiple disabilities, aggressive behavior.

\section{Introduction}

Multiple disabilities are intense and complex combinations of disorders in motor, sensory, linguistic, intellectual and social functioning. The frequent manifestations of challenging behaviors are among the typological characteristics of individuals with multiple disabilities. A hypothesis is raised about the direct dependence of the extreme or unacceptable behavioral problems on the disturbed integration of the information coming through different sensory channels. The choice of an appropriate therapeutic solution for both parents and specialists is sometimes very difficult due to the complex clinical picture of the disorders. A common practice in educational structures is the application of therapeutic methods whose philosophy accepts the behavioral understanding of the child's development: Applied behavioral analysis, Structured Learning method, TEACCH therapy, etc. However, the specified strategies are not far from exhausting the full range of tools used to regulate behavior within the classroom and the school. Ayres' theoretical legacy $(1972,1979)$ have stimulated a number of experimental psychological studies that have revised the resources of sensory-integration therapy (SIT), designed to improve behavioral and emotional functioning. This study is provoked by the author's scientific interest in revealing the positive potential of a specific type of therapy that meets the behavioral needs of children with multiple disabilities.

\section{Literature review}

Aggressive and socially unacceptable human behavior is a topic increasingly discussed in the scientific space. In many studies, dedicated to the problems of special education, the fact of the dominant cases of aggression in individuals with atypical development has manifested (Farmer \& Aman, 2009). The wide range of negative and destructive effects of aggressive behavior is among the widely discussed issues in the modern psychological literature (Barchia \& Bussey, 2011). Low levels of psychosocial adaptation make children with multiple disabilities particularly vulnerable to aggression and violence, which threatens their normal functioning and quality of life. Knowledge of these specifics by the specialists presupposes maximum realization of diagnostic and special training programs. The opportunity to benefit from proven effective therapies to reduce various forms of aggression gives new perspectives in the social and academic functioning of children with different variants of combined disorders.

The contemporary researchers in the psychology of disorders have reviewed and systematized the scientific facts for overcoming or dealing with aggressive behavior, a serious phenomenon that causes negative psychological, social and educational consequences. Therapeutic models developed over the years are characterized mainly by behavioral orientation, which postulates the idea of operant conditioning (Johnson \& Baumeister, 1978; Mason \& Iwata, 1990). Data on other therapies and their

\footnotetext{
${ }^{1}$ Trakia University, Faculty of Education, Stara Zagora, Bulgaria, paskaldi1929@abv.bg
} 
beneficial effects on aggressive behavior are also found in the literature. Theoretical-experimental generalizations refer mainly to developmental (relationship-based) interventions (Atchison et al., 1997) or to physiological models of treatment (Lautenschlager et al., 2012; Eyre \& Baune, 2012). However, the registered empirical information is not sufficient to support the effectiveness of these therapies in children with atypical development (Mason \& Iwata, 1990). In the scientific field, the greatest interest among these intervention models is aroused by sensory-integration therapy (SIT). The fundamental basis of SIT is the theory of sensory integration. The active research on sensoryintegrative therapy began with the original work of Ayres Sensory Integration and the Child (1972). The author defines it as a process that takes place at all levels of the central nervous system and allows the organization, filtering, and interpretation of information coming through all sensory channels. The unique process ensures the adaptive reaction of the individual to the specific environmental situation and forms the basis for theoretical training and social behavior (Tsvetkova, 2015; May-Benson \& Koomar, 2010). The studies on the effectiveness of SIT provide a reason for leading researchers in this field (Wilbarger \& Wilbarger, 1991; Bright et al., 1981; Ayres, 1972; 1979) to determine the significant benefits of its application: (1) increasing the ability to concentrate on the therapeutic process itself, educational materials, social events; (2) a minimization of auto-aggressive manifestations; (3) overall improvement in cognitive functioning, which relates primarily to language and reading ability - a consequence of improved functions of the central nervous system.

The data generated by various studies (Ardent et al., 1988; Vargas \& Camilli, 1999; Faramarzi et al., 2016; Brosnan \& Healy, 2011; Umeda \& Deitz, 2011; Farahiyah et al., 2015; Lang et al., 2012) reflect the authors' discussions regarding the therapeutic effect of the sensory-integrative model on different types of aggressive behavior. The creation of this article is motivated by the contradictory scientific information about the effectiveness of the sensory-integrative therapeutic method in relation to aggressive behaviors in children with multiple disorders, as well as the dynamic discoveries in this field of modern knowledge. The main aim of the study is to establish the effects of sensory-integration therapy on types of aggressive behavior demonstrated by children with multiple disabilities.

\section{Data and Methodology}

The content of the main research thesis is related to the existence of a difference in the frequency of aggressive behaviors of children with multiple disabilities who underwent sensory-integration therapy compared to the behavioral patterns of the same children before its application.

The following null hypotheses were used for this study:

- $\mathbf{H}_{0}$ 1: The difference between the mean levels of aggression towards property noticed in children with more than one developmental disorder placed in contrasting experimental positions (lack - presence of a properly organized sensory environment) will not be characterized by a high significance value.

- Ho2: The difference between the mean levels of verbal aggression registered in the participants positioned in different evaluation conditions (before - after the therapeutic sessions) will not be characterized by a high significance value.

- $\mathbf{H}_{0}$ 3: The difference between the mean levels of physical aggression toward others established in children with multiple disabilities before and after application of the sensory-integrative model of therapy will not be characterized by a high significance value.

- $\mathbf{H}_{0} 4$ : The difference between the mean levels of auto-aggression identified in children with multiple disabilities placed in opposite experimental conditions (before - after the therapeutic sessions) will not be characterized by a high significance value.

The sample was selected by a convenience sampling method. It included 18 children (13 male and 5 female) trained in a special educational institution. All participants had the following multiple disabilities: Emotionally Disabled and Attention Deficit Hyperactivity Disorder, Autism Spectrum Disorder and severe to profound intellectual disabilities, and Down's syndrome. This study used an adapted-time series design (Gall, Gall, \& Borg, 2007). The independent variable was the presence in an organized sensory-therapeutic environment and the dependent variable was the number of negative behavioral responses registered in the four sub-areas of aggression before and after the application of sensory-integration therapy. The data were organized by sub-scores for analysis and reporting in relation to the null hypotheses. The instrument used in this study was a Modified Overt Aggression 
Scale (MOAS) which is considered to be both valid and reliable. The following methods were used in the study: Paired samples t-tests to test the four null hypotheses; a Shaprio-Wilk test for the assumption of normality, and Cohen's d to determine the effect size.

\section{Results}

The aim of this experimental study was to establish the effects of sensory-integration therapy on the number and type of aggressive behaviors exhibited by children with multiple disabilities educated in special educational institutions. The aggressive behavioral patterns of the participants on all four areas (property, verbal, physical, and self) were measured and the results for each of the null hypotheses were presented.

With respect to the first sub-area of study, aggression towards property the results of the Shapiro-Wilk test was not significant ( $p>.05)$ for both control and treatment groups. These results suggest that the assumption of normality in both groups were met. The parametric analysis (a paired samples t-test) of the data showed that after the application of sensory intervention, the mean values of this type of aggressive behavior showed a convincing decrease in the treatment group $(\mathrm{p}<0.01)$. This was a reason to reject the first null hypothesis.

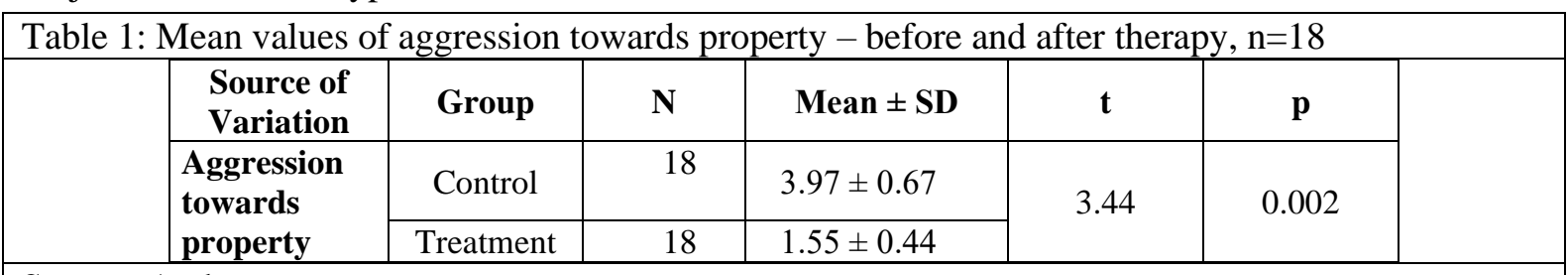

Source: Authors

The total number of challenging behaviors in the control group $(\mathrm{M}=3.97, \mathrm{SD}=0.67)$ and treatment group $(\mathrm{M}=1.55, \mathrm{SD}=0.44)$ were compared and Cohen's effect size value $(\mathrm{d}=1.5)$ suggested a high practical significance. The SIT phase showed a stable low trend of aggressive behavior towards property.

In the study of verbal aggression, a different tendency emerged. The results of the Shapiro- Wilk test was significant $(p<.05)$ for both control and treatment groups These results suggest that the assumption of normality for both the control and treatment groups were not met. However, the t test was determined to be robust enough to handle the violation of normality.

\begin{tabular}{|c|c|c|c|c|c|c|}
\hline & $\begin{array}{l}\text { Source of } \\
\text { Variation } \\
\end{array}$ & Group & $\mathbf{N}$ & Mean \pm SD & $\mathbf{t}$ & $\mathbf{p}$ \\
\hline & \begin{tabular}{|l|} 
Verbal \\
aggression \\
\end{tabular} & $\begin{array}{c}\text { Control } \\
\text { Treatment } \\
\end{array}$ & $\begin{array}{l}18 \\
18 \\
\end{array}$ & $\begin{array}{l}4.48 \pm 3.67 \\
3.03 \pm 2.59 \\
\end{array}$ & 2.05 & 0.05 \\
\hline
\end{tabular}

Source: Authors

The result of the paired samples $t$-test was not significant $(\mathrm{p}=0.05)$. The comparison of the data did not show the existence of statistically significant differences between the two groups (Table 2). Therefore, the second null hypothesis was accepted.

For the second sub-area of research the total number of verbally aggressive behavioral patterns before therapy $(\mathrm{M}=4.482, \mathrm{SD}=3.67)$ and after applying sensory support $(\mathrm{M}=3.03, \mathrm{SD}=2.59)$ were compared and Cohen's effect size value $(\mathrm{d}=0.05)$ suggested no practical significance. As can be seen, the data presented a different trend then the previous domain of study. A slight decrease was found in the frequency of this type of adverse behavior.

For the third variable, the results of the Shapiro- Wilk test was not significant $(\mathrm{p}>.05)$ for both control and treatment groups which is evidence of a normal distribution. The conclusion for a statistically significant reduction and strong elimination of the initial levels of physical aggression toward others when applying the SIT was confirmed by the comparison of the mean values presented in Table 3 ( $\mathrm{p}<$ 0.05). The data showed significant efficiency of the applied technology that led to the rejection of the third hypothesis. 


\begin{tabular}{|c|c|c|c|c|c|c|}
\hline & $\begin{array}{l}\text { Source of } \\
\text { Variation }\end{array}$ & Group & $\mathbf{N}$ & Mean \pm SD & $\mathbf{t}$ & $\mathbf{p}$ \\
\hline & \multirow{2}{*}{\begin{tabular}{|l|} 
Physical \\
aggression \\
toward \\
others
\end{tabular}} & Control & 18 & $3.03 \pm 2.59$ & \multirow[b]{2}{*}{2.33} & \multirow[b]{2}{*}{0.02} \\
\hline & & Treatment & 18 & $1.54 \pm 2.42$ & & \\
\hline
\end{tabular}

Source: Authors

The total occurrences of physical aggression toward others across baseline $(\mathrm{M}=3.03, \mathrm{SD}=2.59)$ and Sensory integration therapy $(\mathrm{M}=1.54, \mathrm{SD}=2.42)$ were compared and Cohen's effect size value ( $\mathrm{d}$ $=1.4$ ) suggested a large practical significance. The strong reduction was seen for the frequency of socially unacceptable behavior demonstrated by children receiving sensory integrative support.

Prior to the analysis of the data in the fourth sub-area, the assumption of normality was assessed. The results of the Shapiro- Wilk test was significant $(\mathrm{p}<.001)$ for both control and treatment groups. Consequently, the assumption of normality in both groups was not met. At the same time, the statistically significant difference in the values obtained before and after the application of the sensory-integrative therapy makes an impression $(\mathrm{p}<0.001)$. The fourth null hypothesis was rejected.

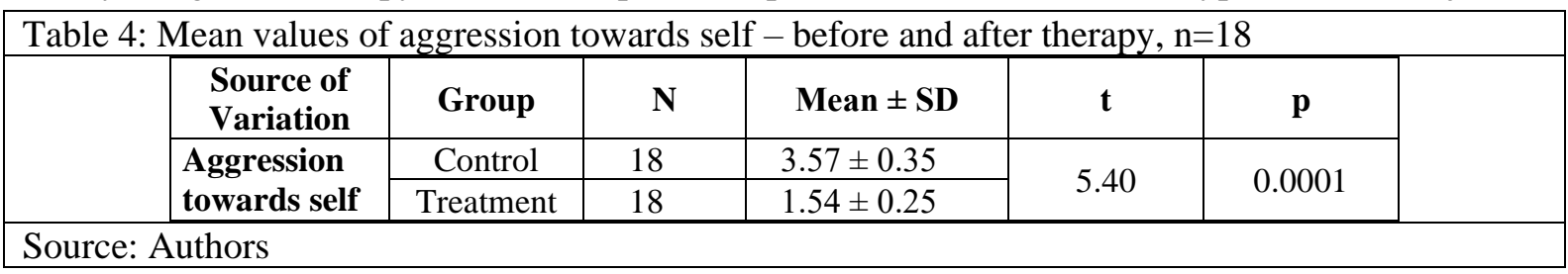

The total number of self-injurious behaviors across output level $(\mathrm{M}=3.57, \mathrm{SD}=0.35)$ and sensory integrative intervention $(\mathrm{M}=1.54, \mathrm{SD}=0.25)$ were compared and Cohen's effect size value $(\mathrm{d}=1.9)$ suggested a large practical significance. The results indicated a strongly decreasing trend in frequency of aggression towards self during the phase including sensory integrative techniques.

\section{Discussion}

The aim of this exploration was to make a direct experimental test on the effectiveness of the used sensory-therapeutic model to reduce the level of aggression in children with more than one developmental disorder. Directly applied monitoring of behavior change and, accordingly, the collection of empirical data provided the most accurate measure of change in behavioral patterns.

The formulated hypotheses were verified by a specific test in four sub-areas. The four assumptions were related to the statement that a significant difference in the frequency of aggressive manifestations would not be registered in the same children placed in different conditions: presence in a specially organized therapeutic environment and lack of therapeutic sessions. The results obtained from the four investigated sub-areas (property, verbal, physical, and self) formed a tendency to minimize the aggressive behavior of the participants in the experiment during the second twelve days of the provided sensory stimulation.

The first hypothesis tested was with a focus on zero effect verification between demonstrated levels of aggression towards the items found in children with multiple disabilities before and after the sensoryintegrative intervention. The established difference was characterized by strong statistical significance therefore, this null hypothesis was rejected. The data provided by the survey correspond to previous studies recommending the use of sensory-integrative therapeutic strategies that meet the behavioral needs of children with different combinations of disorders: autism and severe to profound intellectual disabilities (Cox et al., 2009); developmental disabilities (Brosnan, \& Healy, 2011; Umeda \& Deitz, 2011); as well as children with Attention Deficit Hyperactivity Disorder and a comorbid behavioral concern of aggressive behavior (Farahiyah et al., 2015). According to researchers working on SIT problems (Lang et al., 2012), sensory stimuli designed for "internal" sensory systems (tactile, proprioceptive and vestibular systems) are especially suitable for changing the intensity of arousal. The leading trend, which is confirmed by the review of the literature on the efficiency of SIT, is the set of contradictory data that researchers have published in the scientific space (Ardent et al., 1988; Vargas \& Camilli, 1999). At the same time, in the works of investigators, evidence is found for a 
significant improvement in positive social behavior and, respectively, for a strong limitation of destructive behaviors. In contrast, research corps are revealed in which there is no evidence of particular efficacy of the sensory therapy (Hoehn \& Baumeister, 1994; Leong \& Cater, 2008). The current study belongs to the investigations that confirmed the high efficiency of this type of therapy due to the validated statistically significant difference between the control and treatment groups in favor of a reduced incidence of aggressive actions towards property.

The second null hypothesis focused on the total occurrences of verbal aggression in the respondents from the control and treatment groups, located in different experimental conditions - when they were not subject to sensory therapy and when they attended specialized classes in the sensory room. It is not uncommon for children with atypical development to use verbal aggression as an attempt to control the specific situation. In this way, they put pressure on others (parents, teachers, classmates) and make them fear and abide by their rules (Atkin et al., 2002). The registered data indicated different levels of verbal aggression in children from the separate groups. Due to the lack of a highly significant value of the difference obtained, the pre-formulated null hypothesis was not rejected. These findings are consistent with data from other studies showing that the use of sensory therapy is mainly associated with improved attention and the minimization of constantly recurring patterns of behavior (Fertel-Daly et al., 2001; Losinski et al., 2017), autoaggressive (Doughty \& Doughty, 2008), and destructive behaviors (Quigly et al., 2011). Consequently, in this case it cannot be argued that the application of sensory-based intervention had a serious effect in reducing the frequency of verbal aggression in children with combined disorders. Obviously, the application of SIT does not lead to a high level of emotional and behavioral self-regulation in all subjects. When interpreting the data, the probability that the children's problems are caused by other factors should be taken into account. The number of previous studies in which there are controversial data on the positive effects of sensory-integrative therapy dominates. An explanation for such a trend can be found in the violated requirements for normal distribution due to the limited selection of units in the samples. The current study is certainly no exception to this fact, which is considered a major limitation $(n=18)$. In this context, the assertion of Stevens (2009) is is completely acceptable that if the sample size is characterized by a large enough range (more than 50 units) it will display an imperceptible influence of the deviations from the normality on the final results.

According to the third null hypothesis, the difference between the average values of physical aggression toward others registered in children with multiple disabilities before and after their participation in therapeutic sessions, is not presented with a high level of significance. Undoubtedly, in this way the children have sought attention to meet his/her needs simply because they have not taught in any other way how to do it. It is possible that the aggression has been the result of a painful situation for the child, that he/she has wanted to get out of and it has served as a means of protection. Compared to the control group, the participants in the treatment group remarkably limited the negative patterns typical of one of the forms of unacceptable behavior - aggression towards others. The difference registered with a high level of significance determined this null hypothesis to be rejected. The research data corresponds with other studies (Baranek, 2002; Dawson \& Watling, 2000) that have reported positive effects of SIT with this population in relation to specific performances that could be classified as behavior aimed at causing physical pain to another person or generalized violence against people from their direct environment. Two SIT studies also certified the realistic benefits of a therapeutic approach to unacceptable behavioral patterns for children and adults (Larrington, 1987; Stagnitti et al., 1999). Based on their evidence the researchers have proposed structured SIT programs to deal with the physical aggression of adolescent children. These findings are confirmed by MayBenson and Kumar (2010) through a large-scale investigation to identify the usefulness of the therapeutic model for improving sensory-integrative dysfunction in children. In addition to behavioral, psychological and emotional aspects, the authors summarize the positive effects of regulated sensory stimulation in a broader context - socialization. Overall, the exploration of the literature has shown that heterogeneous results suggest research that needs methodologically, and theoretically precise concepts related to representative samples, fidelity to intervention, selection and interpretation of scores.

The negative assumption in the fourth hypothesis reflects the absence of significant positive influence of the studied therapeutic model on the respondents manifesting aggression towards themselves. 
Difficulties in communicating their own wants and needs; sensory sensitivities, like an oversensitivity to noise or a need for stimulation, and high levels of stress and anxiety are among the leading causes of auto-aggression. An in-depth analysis of behavioral responses in this assessment subzone revealed that significantly lower level of aggression toward oneself were predominant for the treatment group. As result, this null hypothesis was rejected. The findings of this study highlighted the benefits of appropriately structured sensory components of therapy for overcoming or preventing auto-aggressive behaviors. This is consistent with the conclusive results found in the meta-analysis of 11 sensory integration studies (Baranek, 2002). The review reported positive outcomes in some qualitative explorations that identified SIT as an effective approach to reducing auto-aggressive levels (Bright et al., 1981; Wells \& Smith, 1983). The results from these early examinations were in favor of a significantly reduced iterance of aggressive reactions after classes were organized in conditions of sensory stimulation. Despite the encouraging data in the current study, the findings of systematic literature reviews have testified to a low percentage of evidence in support of the discussed method of therapy in children with combined disorders.

\section{Conclusion}

The present study examined the effectiveness of the application of sensory-integration therapy in children with multiple disabilities who exhibited aggressive behaviors. The registration of children's behavioral responses for 24 consecutive school days allowed the collection of evidence in support of the thesis in the study. During the first 12 days no therapeutic sessions were organized for the children, while during the next 12 days a specialist in prepared sensory-intervention schemes worked with them. Documenting and reporting the behavioral models in the individual protocols of the children covered 4 sub-areas: aggression toward property, verbal aggression, physical aggression toward others, and aggression toward self. The statistical results indicated a high level of significance for the three variables. The obtained average values for three of the evaluated modules are evidence for the positive effects of the sensory-based therapy in children with emotional and behavioral problems. In regard to verbal aggression, no significant difference was reported in the frequency of behavioral reactions before and after the application of specially selected sensory stimulation.

The derived empirical facts allowed us to make a conclusion that the organization of sensory perception and the effective interaction with sensory stimuli helped to overcome aggression and improve behavioral functioning in children. Reducing negative behavior allows the child to give precedence to his/her strengths and successfully cope with daily school and life situations. The positive effects of a well-organized therapeutic environment will be exhibited in the formation of adaptive behavior, development of new skills, and productive responses to difficulties.

The conducted study is an attempt to formulate questions, provide topics for discussion, outline problems motivating new research for specialists, $\mathrm{PhD}$ students and other professionals, involved in the overall development and prosperity of this diverse by age, abilities, interests and experience group of children.

\section{References}

Atchison, K., Bartlebaugh, K., Freeman, B. J., Benavides, M., George, K. \& Guhrman, H. (1997). Best practices for designing and delivering effective programs for individuals with autistic spectrum disorders: Recommendations of the collaborative working group on autistic spectrum disorders, Sacramento, US: Resources in Special Education (RiSE).

Ayres, A. J. (1972). Sensory integration and learning disorders, Los Angeles: Western Psychological Services.

Ayres, A. J. (1979). Sensory integration and the child, Los Angeles: Western Psychological Services.

Arendt, R.E., MacLean, W.E. \& Baumeister, A.A. (1988). Critique of sensory integration therapy and its application in mental retardation, American Journal of Mental Retardation, 92, 401-411.

Atkin, C., Smith, S., Roberto, A., Fediuk, T., Wagner, T. (2002). Correlates of verbally aggressive communication in adolescents, Journal of Applied Communication Research, 30, 251-268.

Baranek, G. T. (2002). Efficacy of Sensory and Motor Interventions for children withAutism, Journal of Autism and Developmental Disorders, 32, 397-422.

Barchia, K. \& Bussey, K. (2011). Predictors of student defenders of peer aggression victims: empathy and social cognitive factors, International Journal of Behavioral Development 35(4) 289-297.

Bright, T., Bittick, K. \& Fleeman, B. (1981). Reduction of self-injurious behavior using sensory-integrative techniques, American Journal of Occupational Therapy, 35, 167-172.

Brosnan, J. \& Healy, O. (2011). A review of behavioural interventions for the treatment of aggression in individuals with developmental disabilities, Research in developmental Disabilities, 32, 437- 446. 
Cox, A. L., Gast, D. L., Luscre, D. \& Ayres, K. M. (2009). The effects of weighted wests on appropriate in-seat behaviors of elementary-age students with autism and severe to profound intellectual disabilities, Focus on Autism and other Developmental Disabilities, 24, 17-26.

Dawson, G. \& Watling, R. (2000). Interventions to facilitate auditory, visual, and motor integration in autism: A review of the evidence, Journal of Autism and Developmental Disorders, 30, 415- 421.

Eyre, H. \& Baune, B. T. (2012). Neuroimmunological effects of physical exercise in depression, Brain, Behavior, and Immunity, 26 (2), 251-266.

Doughty, S. \& Doughty, A. (2008). Evaluation of body-pressure intervention for self-injury in autism, The Behavioral Development Bulletin, 14, 23-29.

Farahiyah W, Liu, K., Bissett, M., Penkala, S. (2015). Sensory based intervention for children with behavioral problems. A systematic review, Journal of Autism and Developmental Disorders, 45, 3565-3579.

Faramarzi, S., Arjmandi, A. \& Abedi, A. (2016). Effect of sensory integration training on executive functions of children with attention deficit hyperactivity disorder, Neuropsychiatria i Neuropsychologia 11 (1), 1-5.

Farmer, C. \& Aman, M. (2009). Development of the children's scale of hostility and aggression: Reactive/Proactive (CSHARP), Research in Developmental Disabilities, 30, 1155-1167.

Fertel-Daly, D., Bedell, G. \& Hinojosa, J. (2001). Effects of a weighted vest on attention to task and self-stimulatory behaviors in preschoolers with pervasive developmental disorders, American Journal of Occupational Therapy, 55, 629-640. Gall, M., Gall, J. \& Borg, W. (2007). Educational research: An introduction (8th ed.), New York, NY: Allyn \& Bacon. Hoehn, T. P. \& Baumeister, A. A. (1994). A critique of the application of sensory integration therapy to children with learning disabilities, Journal of Learning Disabilities, 27, 338-350.

Johnson, W. L. \& Baumeister, A. (1978). Self-injurious behavior: A review and analysis of methodological details of published studies, Behavior Modification, 2, 465-485.

Lang, R., O’Reilly, M., Healy, O., Rispoli, M., Lydon, H., Streusand, W., Davis, T., Kang, S., Sigafoos, J., Lancioni, G, Didden, R. \& Giesbers, S. (2012). Sensory integration therapy for autism spectrum disorders: A systematic review, Research in Autism Spectrum Disorders, 6, 1004-1018.

Larrington, G. G. (1987). A sensory integration based program with a severely retarded/autistic teenager: An occupational therapy case report. In Z. Mailloux (Ed.), Sensory integration approaches (pp. 101-117), New York: Hawthorn Press.

Lautenschlager, N. T., Cox, K. \& Cyarto, E. V. (2012). The influence of exercise on brain aging and dementia, Biochimica et Biophysica Acta (BBA)-Molecular Basis of Disease, 1822 (3), 474-481.

Leong, H. M. \& Carter, M. (2008). Research on the efficacy of sensory integration therapy: Past, present and future, Australasian Journal of Special Education, 32, 83-99.

Losinski, M., Cook, K., Hirsch, S. \& Sanders, S. (2017). The effects of deep pressure therapies and antecedent exercise on stereotypical behaviors of students with autism spectrum disorders, Behavioral Disorders, 42 (4), 196-208.

Mason, S. A. \& Iwata, B. A. (1990). Artifactual effects of sensory-integrative therapy on self-injurious behavior, Journal of Applied Behavior Analysis, 23, 361-370.

May-Benson, T. A. \& Koomar, J. A. (2010). Systematic review of the research evidence examining the effectiveness of interventions using a sensory integrative approach for children, American Journal of Occupational Therapy, 64, 403-414.

Schaffer, R. (1984). Sensory integration therapy with learning disabled children: A critical review, Canadian Journal of Occupational Therapy, 51, 73-77.

Stagnitti, K., Raison, P., Ryan, P. (1999). Sensory defensiveness syndrome: A paediatric perspective and case study, Australian Occupational Therapy Journal, 46, 175-187.

Stevens, J. P. (2009). Applied multivariate statistics for the social sciences (5th ed.), Mahwah, NJ: Routledge Academic. Tsvetkova-Arsova, M. (2015). Pedagogy of children and students with multiple disabilities, Publishing house, Phenomenon. $\mathrm{S}$.

Umeda, C. \& Deitz, J. (2011). Effects of therapy cushions on classroom behaviors of children with autism spectrum disorder, American Journal of Occupational Therapy, 65, 152-159.

Vargas, S. \& Camilli, G. (1999). A meta-analysis of research on sensory integration treatment, The American Journal of Occupational Therapy, 53, 189-198.

Wells, M. \& Smith, D. W. (1983). Reduction of self-injurious behavior of mentally retarded persons using sensory integrative techniques, American Journal of Mental Deficiency, 87, 664-666.

Wilbarger, P. \& Wilbarger, J. (1991). Sensory defensiveness in children aged 2-12: An intervention guide for parents and caregivers, Denver, CO: Avanti Educational Programs. 